\title{
A novel framework of tissue membrane systems for image fusion
}

\author{
Zulin Zhang ${ }^{\mathrm{a}}$, Xinzhong $\mathrm{Yi}^{\mathrm{b}, *}$ and Hong Peng ${ }^{\mathrm{c}}$ \\ ${ }^{a}$ Department of Computer Science, Sichuan University for Nationalities, Kangding 626001, China \\ ${ }^{\mathrm{b}}$ Department of Finance and Management, Leshan Vocational and Technical College, Leshan 614000, \\ China \\ ${ }^{\mathrm{c}}$ Center of Radio Administrator and Technology Development, Xihua University, Chengdu 610039, \\ China
}

\begin{abstract}
This paper proposes a tissue membrane system-based framework to deal with the optimal image fusion problem. A spatial domain fusion algorithm is given, and a tissue membrane system of multiple cells is used as its computing framework. Based on the multicellular structure and inherent communication mechanism of the tissue membrane system, an improved velocity-position model is developed. The performance of the fusion framework is studied with comparison of several traditional fusion methods as well as genetic algorithm (GA)-based and differential evolution (DE)-based spatial domain fusion methods. Experimental results show that the proposed fusion framework is superior or comparable to the other methods and can be efficiently used for image fusion.
\end{abstract}

Keywords: Membrane computing, membrane systems, tissue membrane systems, image fusion

\section{Introduction}

Usually, it is impossible that all significant objects contained in an image are in focus due to the limited depth-of-focus of optical lenses in imaging sensors. Image fusion is one of the most effective methods that deal with this problem. In order to obtain an image that contains the outlines of the scene as well as significant objects, image fusion can integrate the information provided by various imaging sensors to produce a new and improved single image, known as the fused image. During the last decade, a large number of image fusion methods have been proposed [1]. Among them, an interesting fusion method is based on the selection of sharper image blocks from source images. However, the existing works have shown that block size can significantly affect the fusion results. Therefore, it is important to determine the most appropriate block size for the fusion method, which can be posed as an optimal image fusion problem. However, it is also difficult and time-consuming to search the optimal block sizes in an exhaustive way due to huge combinations of block sizes. Recently, several works have shown

\footnotetext{
*Corresponding author: Xinzhong Yi, Department of Finance and Management, Leshan Vocational and Technical College, Leshan 614000, China. Tel.: +86-0833-2109414; Fax: +86-0833-2131129; E-mail: ph.xhu@ hotmail.com.
}

0959-2989/14/\$27.50 @ 2014 - IOS Press and the authors. 
that some heuristic optimization algorithms can be used to overcome the above limits, such as genetic algorithms (GA), differential evolution (DE) algorithms. Kong et al. [2] used the GA to explore the optimal block sizes for image blocks in a source image. However, GA has some shortcomings, such as slow convergence rate, premature convergence to local minima, spending much computation time in the encoding and decoding processes of individuals. In order to overcome these problems, Aslantas et al. [3] presented a fusion method, which employed the DE to search the optimal block sizes. The DE-based fusion method has revealed faster convergence speed and better fusion effects compared with the GAbased fusion method. However, the performance of DE is sensitive to the choice of the mutation strategy and the associated control parameters [4].

Membrane computing [5], as a class of distributed parallel computing models, is inspired from the structure and functioning of living cells as well as from the cooperation of cells in tissues, organs and population of cells, known as membrane systems or P systems [6]. Over the past years, a variety of variants of membrane systems have been reported [7-10]. Research results on a lot of NP-hard problems have demonstrated that membrane systems are more powerful and competitive compared with other existing approaches [11-13]. The motivation behind this work is to develop a novel image fusion framework in order to solve the optimal image fusion problem by applying the parallel computing ability and evolutioncommunication mechanism of tissue membrane systems. For this purpose, a fusion framework based on tissue membrane systems is developed in this paper, where a tissue membrane system is designed as its computing framework and an improved velocity-position model is developed to evolve objects in the system.

\section{Methods}

\subsection{An image fusion algorithm}

The image fusion algorithm is a spatial domain image fusion algorithm, which is similar to the fusion algorithms employed in [2] and [3]. The idea of the fusion algorithm is that source images are firstly divided into image blocks, and then the fused image is constructed by choosing the sharper image blocks from the source images. In order to choose the sharper image blocks, a criterion function to evaluate the sharpness of the image blocks is required. A commonly used criterion function, spatial frequency, is employed as an evaluation criterion in this work. For an image (or image block) with size $M_{1} \times M_{2}$, its spatial frequency is computed by

$$
S F=\sqrt{C^{2}+F^{2}}
$$

where $C$ and $F$ are the row and column gradients, respectively, and are defined as follows

$$
C=\left[\frac{1}{M_{1} \times M_{2}} \sum_{i=1}^{M_{1}} \sum_{j=1}^{M_{2}}\left[f_{i, j}-f_{i-1, j}\right]^{2}\right]^{\frac{1}{2}}, R=\left[\frac{1}{M_{1} \times M_{2}} \sum_{i=1}^{M_{1}} \sum_{j=1}^{M_{2}}\left[f_{i, j}-f_{i, j-1}\right]^{2}\right]^{\frac{1}{2}}
$$

where $f_{i, j}$ expresses gray level of the image at the pixel $(i, j), 1 \leq i \leq M_{1}, 1 \leq j \leq M_{2}$. 


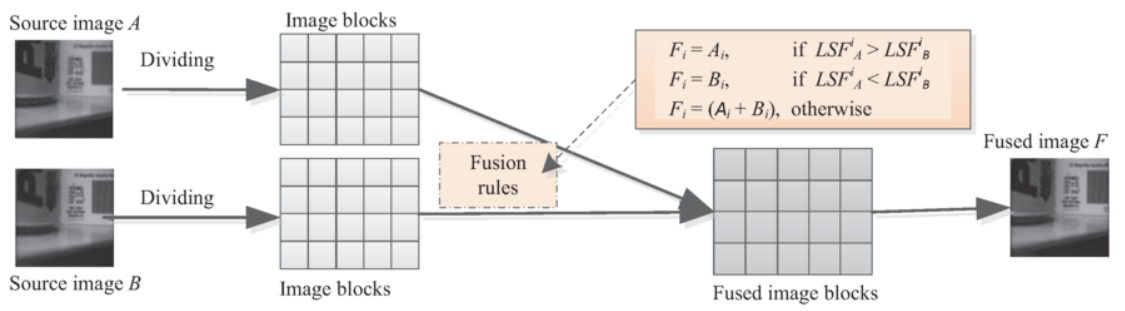

Fig. 1. The image fusion procedure of the fusion algorithm.

Suppose that $A_{i}$ and $B_{i}$ are $i$ th image blocks in source images $A$ and $B$ respectively and their spatial frequencies are denoted by $L S F_{A}^{i}$ and $L S F_{B}^{i}$ respectively. In the present fusion framework, a fitness function is required to evaluate the quality of each object in the tissue membrane system. The spatial frequency of the fused image is used as the fitness value of an object. In other words, source images are partitioned according to the object's components (block size), and then the fusion algorithm is used to form the fused image and the spatial frequency of the fused image is computed as the fitness value of the object. The fitness value of $j$ th object $\vec{x}_{k, j}$ in $k$ th cell is denoted by fitness $s_{k, j}=G S F_{k, j}$.

Figure 1 shows the image fusion procedure of the fusion algorithm. The main steps of the image fusion algorithm can be described as follows:

1) Divide the source images $A$ and $B$ into non-overlapping image blocks according to the parameter (block size) of the current object $\vec{x}_{k, j}$. Let $A_{i}$ and $B_{i}$ be $i$ th image blocks of source images $A$ and $B$, respectively.

2) Compute the sharpness values of image blocks $A_{i}$ and $B_{i}, L S F_{A}^{i}$ and $L S F_{B}^{i}$, respectively.

3) Construct $i$ th image block $F_{i}$ of the fused image according to the following fusion rules:

$$
F_{i}= \begin{cases}A_{i}, & \text { if } L S F_{A}^{i}>L S F_{B}^{i} \\ B_{i}, & \text { if } L S F_{A}^{i}<L S F_{B}^{i} \\ \left(A_{i}+B_{i}\right) / 2, & \text { otherwise }\end{cases}
$$

4) After employing Eq. (3) for image blocks of source images, compute the spatial frequency of the fused image as the fitness value of the current object $\vec{x}_{k, j}$, fitness $s_{k, j}=G S F_{k, j}$.

\subsection{Image fusion framework based on tissue membrane systems}

Tissue membrane systems are a kind of variants of membrane systems, which are inspired from the behaviours of several one-membrane cells evolved in a common environment [14]. A tissue membrane system can be viewed as a net of processors, which can deal with the objects and communicate them according to the channels given in advance. In this work, a tissue membrane system consisting of $m$ cells is considered as a computing framework, which is defined as the following construct:

$\Pi=\left(X_{1}, \ldots, X_{m}, R_{1}, \ldots, R_{m}, R^{\prime}, i_{o}\right)$

where

1) $X_{i}$ is the set of $N$ objects in cell $i, 1 \leq i \leq m$;

2) $R_{i}$ is the evolution rule in cell $i$, which is an improved position-velocity model, $1 \leq i \leq m$; 


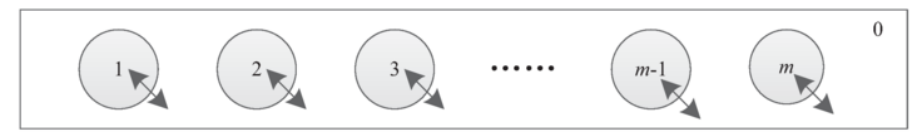

Fig. 2. The tissue membrane system of $m$ cells.

3) $R^{\prime}$ is a finite set of communication rules of the form $(i, \vec{x} / \lambda, 0), i=1,2, \ldots, q, \lambda$ is the empty object;

4) $i_{o}$ indicates the output region of the system.

Figure 2 shows the tissue membrane system of $m$ cells. The $m$ cells are labeled by $1,2, \ldots, m$ respectively, while the environment is denoted by 0 . Usually, each cell contains several objects and evolution rules. The role of evolution rules is to evolve the objects in cells. The present fusion framework searches the optimal fusion parameters by the evolution of objects in cells. Assume that each cell has $N$ objects, while the environment has only one object which is the best object to be found so far (called the global best object). In the fusion framework, each object is a two-dimensional vector $\vec{x}=\left(x_{1}, x_{2}\right)$, where $x_{1}$ and $x_{2}$ are width and height of an image block respectively.

In the fusion framework, an improved position-velocity model is developed according to the multicellular structure and inherent communication mechanism of the tissue membrane system, which can be viewed as a variant of the position-velocity model in particle swarm optimization (PSO). For $j$ th object of $k$ th cell, $\vec{x}_{k, j}$, the improved position-velocity model can be described as follows.

$$
\begin{aligned}
& \vec{v}_{k, j}=\omega \cdot \vec{x}_{k, j}+c_{1} r_{1}\left(\vec{p}_{k, j}-\vec{x}_{k, j}\right)+c_{2} r_{2}\left(\vec{g}_{k}-\vec{x}_{k, j}\right)+c_{3} r_{3}\left(\vec{g}_{*}-\vec{x}_{k, j}\right), \\
& \vec{x}_{k, j}=\operatorname{floor}\left(\vec{x}_{k, j}+\vec{v}_{k, j}\right), \quad j=1,2, \ldots, N, k=1,2, \ldots, m .
\end{aligned}
$$

where $\omega$ is inertia weight, $c_{1}, c_{2}$ and $c_{3}$ are learning factors, $r_{1}, r_{2}, r_{3} \in(0,1)$ are random numbers, and floor $(\cdot)$ is a standard rounded function. $\vec{x}_{k, j}=\left(x_{k, j, 1}, x_{k, j, 2}\right)$ expresses the position of the object $\vec{x}_{k, j}$ in solution space, $\vec{v}_{k, j}=\left(v_{k, j, 1}, v_{k, j, 2}\right)$ is the corresponding velocity vector, and $\vec{p}_{k, j}$ is the best position of the object $\vec{x}_{k, j}$ to be found so far. The improved position-velocity model uses two best objects to guide the evolution of objects in each cell: one is the best object in current cell, $\vec{g}_{k}=\left(g_{k, 1}, g_{k, 2}\right)$, and another is the global best object in all cells to be found so far, $\vec{g}_{*}=\left(g_{* 1}, g_{* 2}\right)$. As a result, the consideration can bring two advantages: (1) convergence acceleration; (2) diversity improvement.

In the present tissue membrane system, there are some communication channels of objects between cells and the environment, shown by the arrows in Figure 2, which are realized by communication rules. After all objects are evolved, each cell will communicate its best object into the environment and update the global best object. The update rule used is as follows: if the fitness value of the communicated object is higher than that of existing object in the environment, it will replace the existing object; otherwise, the communicated object is discarded.

As usual in membrane systems, all of the cells as parallel computing units work in a maximally parallel way (a universal clock is considered here). The environment is considered to be the output region of the system. The maximum execution step number is employed as halting condition in the fusion framework. When the system halts, the global best object in the environment will be regarded as the final computing result, namely, the optimal block size.

The main steps of the proposed fusion framework can be described as follows: 
1) Randomly generate $N$ initial objects for each cell.

2) For each cell $k(k=1,2, \ldots, m)$, the following steps are executed (all cells work in parallel):

a) Communicate the global best object $\vec{g}_{*}$ into cell $k$ from the environment.

b) For each object $\vec{x}_{k, j}(j=1,2, \ldots, N)$ in cell $k$, repeatedly execute the following evolution operation of objects:

i) Use the position-velocity model (4-5) to evolve the object $\vec{x}_{k, j}$.

ii) According to the new parameter (block size), $\vec{x}_{k, j}=\left(x_{k, j, 1}, x_{k, j, 2}\right)$, call the spatial domain image fusion algorithm described in Section 2.1 to form the fused image, and then calculate the corresponding fitness value.

iii) Update the best position $\vec{p}_{k, j}$ of the current object.

c) After all objects in cell $k$ are evolved, update its best object, $\vec{g}_{k}$.

3) When all objects in all cells are evolved, their best objects are communicated to update the global best object in the environment.

4) Repeat the steps 2) - 3) until a predefined halt condition is satisfied.

\section{Experimental results}

In experiments, input parameters of the proposed fusion framework are given as follows: (1) the tissue membrane system used includes five cells $(m=5)$, where the number of objects contained in each cell is $N=20$, and the maximum execution step is 100; (2) in the improved position-velocity model, $c_{1}=c_{2}=c_{3}=1.0$, and $\omega$ linearly varies from 0.9 to 0.4 .

In order to evaluate the availability and effectiveness of the proposed framework, four test images widely used in literature are considered, including "pepsi", "toy", "lab" and "disk", shown in Figure 3. Figures 3(a")-(d") show the fusion results of the proposed framework on the four images, respectively. From the visual point of view, the proposed fusion framework obtains a satisfactory fusion effect.

For comparison purpose, the fusion experiments are also performed using other five fusion methods, including Laplacian pyramid method, discrete wavelet transform (DWT)-based and contourlet transform (CT)-based methods as well as GA-based and DE-based methods, as shown in Figure 4. For Laplacian pyramid and DWT-based methods, four-level decomposition is employed, and then 'db4' wavelet is used in DWT-based method. For CT-based method, four-level decomposition with 4, 8, 8, 16 directions from coarser scale to finer scale is used. The GA-based and DE-based methods use the same fusion algorithm as that in the proposed fusion framework. The parameters of GA-based method are given: population size $N P=20$, crossover probability $P_{c}=0.6$, mutation rate $P_{m}=0.01$ and maximum generation number $t_{\max }=100$. The parameters of DE-based method are given: population size $N P=20$, crossover probability $P_{c}=0.6$, scalar factor $F=0.9$ and maximum generation number $t_{\max }=100$.

In order to provide a quantitative comparison among different fusion methods, three commonly used metrics, namely, mean square error (MSE), difference coefficient (DC) and mutual information (MI), are used to evaluate the fusion performance. The MSE and DC are, respectively, defined as

$$
\operatorname{MSE}=\frac{1}{M_{1} \times M_{2}} \sum_{i=1}^{M_{1}} \sum_{j=1}^{M_{2}}[R(i, j)-F(i, j)]^{2}, \quad \mathrm{DC}=\frac{1}{M_{1} \times M_{2}} \sum_{i=1}^{M_{1}} \sum_{j=1}^{M_{2}} \frac{|R(i, j)-F(i, j)|}{R(i, j)}
$$




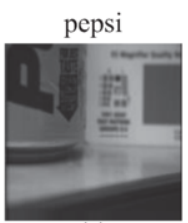

(a)

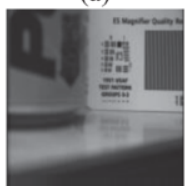

$\left(a^{\prime}\right)$

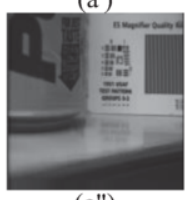

(a")

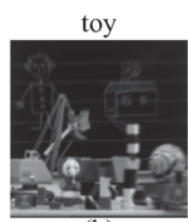

(b)

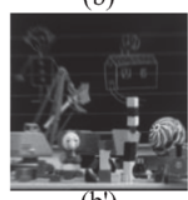

$\left(b^{\prime}\right)$

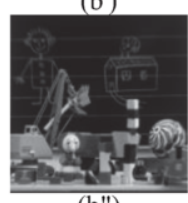

(b")

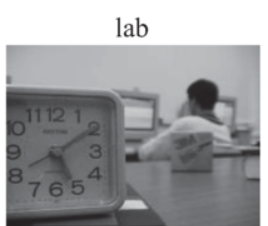

(c)

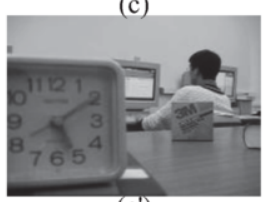

$\left(c^{\prime}\right)$

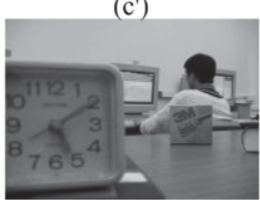

$(\mathrm{c} ")$
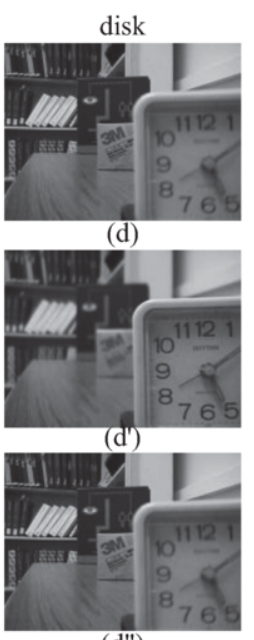

(d")

Fig. 3. The source images ((a)-(d) focus on left part; (a')-(d') focus on right part and fusion results of the proposed fusion framework ((a")-(d")).

Table 1

The comparison results of different fusion methods on "pepsi" image.

\begin{tabular}{lllll}
\hline Fusion methods & MSE & DC & MI & \\
\hline Tissue membrane systems & $4.6752( \pm 0.068)$ & $0.0224( \pm 0.002)$ & $2.4823( \pm 0.054)$ \\
DE & $4.7839( \pm 0.073)$ & $0.0237 \quad( \pm 0.005)$ & $2.4785( \pm 0.062)$ \\
GA & $5.0352( \pm 0.394)$ & $0.0285( \pm 0.015)$ & $2.4634( \pm 0.218)$ \\
Laplacian pyramid & 17.3724 & 0.0859 & 2.3975 \\
DWT & 9.2361 & 0.0458 & 2.4139 \\
CT & 4.9926 & 0.0257 & 2.4586 \\
\hline
\end{tabular}

where $R(i, j)$ and $F(i, j)$ are the pixel values of the reference image and the fused image respectively, and $M_{1} \times M_{2}$ is the image size. The mutual information (MI) between the source images $A, B$, and the fused image $F$ is defined as

$$
\mathrm{MI}=\mathrm{MI}_{A F}+\mathrm{MI}_{B F}
$$

where the mutual information $\mathrm{MI}_{A F}$ between the source images $A$ and $F$ is given as follows

$$
\mathrm{MI}_{A F}=\sum_{a, f} p_{A F}(a, f) \log \frac{p_{A F}(a, f)}{p_{A}(a) p_{F}(f)}
$$

where $p_{A F}(a, f)$ is the jointly normalized histogram of $A$ and $F, p_{A}(a)$ and $p_{F}(f)$ are the normalized histograms of $A$ and $F$ respectively. The mutual information $\mathrm{MI}_{B F}$ between the source images $B$ and $F$ is similar to $\mathrm{MI}_{A F}$. The smaller the values of MSE and DC, the better the fused image, however, the larger the MI value, the better the fusion result. 


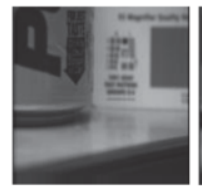

(a)

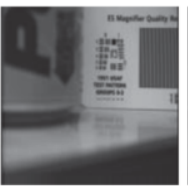

(b)

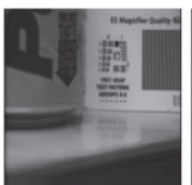

(c)

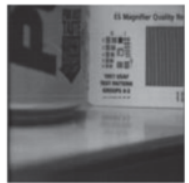

(d)

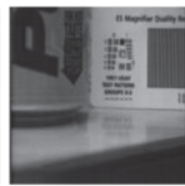

(e)

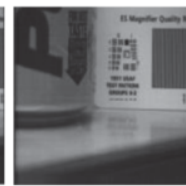

(f)

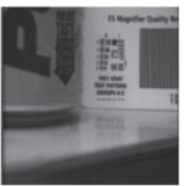

(g)

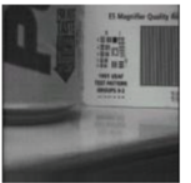

(h)

Fig. 4. The 'pepsi' source images and fusion results of different fusion methods: (a) focus in the left; (b) focus in the right; (c) the proposed fusion framework; (d) Laplacian pyramid method; (e) DWT-based method; (f) CT-based method; (g) GA-based method; (h) DE-based method.

Only the fusion results on "pepsi" image are reported due to the space limit of this paper. Figure 4 gives the source images and the fusion results obtained by the proposed fusion framework and other five fusion methods. It can be seen from these visual comparison results that the proposed fusion framework and the DE-based method have better fusion results compared with other fusion methods. By employing the MSE, DC and MI metrics, quantitative comparison results of these fusion methods are presented in Table 1. Since the proposed fusion framework, GA-based and DE-based fusion methods are stochastic algorithms, the fusion results of the three methods are the mean and standard deviations of MSE, DC and MI results of the experiments repeating 20 times, respectively.. These results show that the proposed fusion framework is slightly better than the DE-based fusion method but obviously superior than other fusion methods. Therefore, these comparison results clearly demonstrate the effectiveness of the proposed fusion framework. In addition, it can be viewed from Table 1 that the proposed fusion framework has the lowest standard deviations compared with the DE-based and GA-based fusion methods. This fact illustrates that the proposed fusion framework is robust.

\section{Conclusion and future work}

This paper has described a fusion framework based on tissue membrane systems, which can exploit the optimal block size for an image fusion algorithm in spatial domain. The experimental results have demonstrated that the proposed fusion framework outperforms the other methods in terms of computation efficiency and robustness. However, the fusion framework is only limited to the image fusion in spatial domain, so our further work will focus on using membrane systems to develop the fusion framework in transform domain, for example, contourlet transform or non-subsampled contourlet transform.

\section{Acknowledgment}

This work was partially supported by the National Natural Science Foundation of China (No. 61170030), Chunhui Project Foundation of the Education Department of China (No. Z2012025, No. Z2012031), Research Fund of Sichuan Key Technology Research and Development Program (No. 2013GZX0155), China.

\section{References}

[1] A.A. Goshtasby and S. Nikolov, Image fusion: advances in the state of the art, Information Fusion 8 (2007), 114-118. 
[2] J. Kong, K. Zheng, J. Zhang and X. Feng, Multi-focus image fusion using spatial frequency and genetic algorithm, International Journal of Computing Science and Network Security 8 (2008), 220-224.

[3] V. Aslantas and R. Kurban, Fusion of multi-focus images using differential evolution algorithm, Expert Systems with Applications 37 (2010), 8861-8870.

[4] R. Mallipeddi, P.N. Suganthana, Q.K. Panb and M.F. Tasgetirenc, Differential evolution algorithm with ensemble of parameters and mutation strategies, Applied Soft Computing 11 (2011), 1679-1696.

[5] Gh. Păun, Computing with membranes, Journal of Computer System Sciences 61 (2000), 108-143.

[6] Gh. Pǎun, G. Rozenberg and A. Salomaa, The Oxford handbook of membrance computing, Oxford University Press, New York, 2010.

[7] Gh. Păun and M.J. Pérez-Jiménez, Membrane computing: brief introduction, recent results and applications, BioSystem 85 (2006), 11-22.

[8] H. Peng, J. Wang, M.J. Pérez-Jiménez, H. Wang, J. Shao and T. Wang, Fuzzy reasoning spiking neural P system for fault diagnosis, Information Sciences, 235 (2013), 106-116.

[9] J. Wang, P. Shi, H. Peng, M.J. Pérez-Jiménez and T. Wang, Weighted fuzzy spiking neural P systems, IEEE Transactions on Fuzzy Systems 21 (2013), 209-220.

[10] H. Peng, J. Wang, M.J. Pérez-Jiménez and P. Shi, A novel image thresholding method based on membrane computing and fuzzy entropy, Journal of Intelligent \& Fuzzy Systems 24 (2013), 229-237.

[11] L. Huang, I. Suh and A. Abraham, Dynamic mul-objective optimization based on membrane computing for control of time-varying unstable plants, Information Sciences 181 (2011), 2370-2391.

[12] G. Zhang, J. Cheng, M. Gheorghe and Q. Meng, A hybrid approach based on different evolution and tissue membrane systems for solving constrained manufacturing parameter optimization problems, Applied Soft Computing 13 (2013), 15281542.

[13] H. Peng, J. Wang, M.J. Pérez-Jiménez and A. Riscos-Núñez, The framework of P systems applied to solve optimal watermarking problem, Signal Processing 101 (2014) 256-265.

[14] R. Freund, Gh. Păun and M.J. Pérez-Jiménez, Tissue-like P systems with channel-states, Theoretical Computer Sci. 330 (2005), 101-116. 